\title{
Erfahrung des kooperativen Brustzentrums Wiesbaden mit §116b SGB V
}

\author{
Jana Barinoffa Rita Hils $^{\mathrm{a}}$ Götz Brodermann ${ }^{\mathrm{b}}$ \\ ${ }^{a}$ Klinik für Gynäkologie und Gynäkologische Onkologie, \\ ${ }^{\text {b } V e r t r a g s m a n a g e m e n t, ~ D r .-H o r s t-S c h m i d t-K l i n i k e n, ~ W i e s b a d e n, ~}$ \\ ${ }^{c}$ Berufsverband der Frauenärzte e.V., Steinbach, Germany
}

Das kooperative Brustzentrum (BZ) Wiesbaden ist ein Teil der Klinik für Gynäkologie und Gynäkologische Onkologie an der Dr.-Horst-Schmidt-Klinik/Wiesbaden. Das Zentrum feiert dieses Jahr sein 5-jähriges Bestehen: 2004 wurde das BZ Wiesbaden als zertifiziertes Brustzentrum durch die Deutschen Krebsgesellschaft und die Deutschen Gesellschaft für Senologie anerkannt. Im gleichen Jahr erfolgte die Akkreditierung durch die EUSOMA und die Positionierung als Referenzzentrum für minimalinvasive Biopsietechniken und Mammasonographie. Neben dem Schwerpunkt in der Diagnostik und im operativen Bereich wird das Versorgungskonzept des BZ Wiesbaden durch das Zentrum für ambulante systemische Therapien (ZAT) ergänzt. Die Studienzentrale, die 2009 durch ein Audit der German Breast Group zum Studienzentrum der Gruppe A zertifiziert worden ist, rundet das Leistungsspektrum des BZ Wiesbaden ab. Die Einführung von klaren Strukturen, die Definition von Arbeitsprozessen und die Pflege des Qualitäts-Management-Systems erforderten anfangs einen hohen Aufwand, führten aber mit der Zeit zu der gewünschten Verbesserung der Leistungsqualität.

Die Erfolgsgeschichte des BZ Wiesbaden reicht jedoch noch weiter zurück. Seit 1998 werden die Daten zur Diagnose «Mammakarzinom» nach bestimmten Kriterien in der Klinik für Gynäkologie und Gynäkologische Onkologie/HSK erfasst. Im Jahr 2002 wurde das Netzwerk mit niedergelassenen

\section{Abb. 1. Netzwerk} Qualitätssicherung Mammakarzinom Wiesbaden: Entwicklung des Netzwerkprojektes Teilnehmende Frauenärzte.

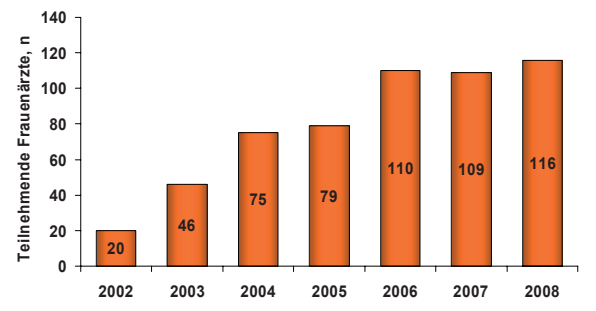

\section{KARGER}

Fax +497614520714

Information@Karger.de

www.karger.com
Frauenärzten zur Qualitätssicherung auf dem Gebiet Mammakarzinom gegründet. Das Ziel dabei war eine lückenlose, Follow-up-pflegende Datenerhebung, die zur Diagnostik- und Therapieoptimierung führen sollte. Es ist gelungen, im Laufe der Zeit über 100 Frauenärzte (Abb. 1) für diese Idee zu gewinnen, und das Ergebnis kann sich sehen lassen: von 91\% der 1998 behandelten Mammakarzinompatientinnen, liegt ein aktueller Status vor, der nicht älter als 11 Monate ist. In 53\% der Fälle erfolgte die Rückmeldung durch Frauenärzte, die restliche Daten wurden durch direkten Kontakt mit den Patientinnen erhoben. Dieses Konzept war ein Meilenstein in der Entwicklung einer vertrauensvollen Zusammenarbeit mit niedergelassenen Kollegen und erlaubte die Etablierung weiterer Projekte von denen wir eines hier präsentieren möchten.

Abb. 2. Erstdiagnose und Therapie in der HSK 1998-2008.

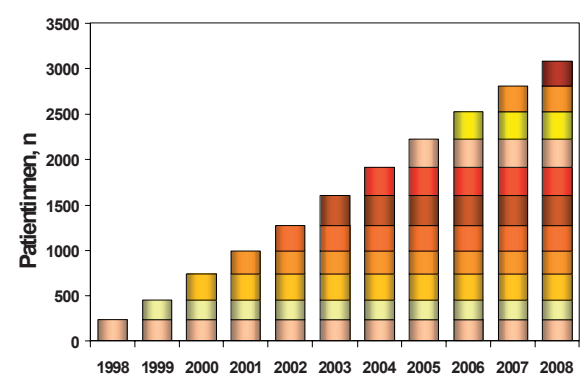

Abb. 3. Erstdiagnose und Therapie in der HSK 1998- 2008.

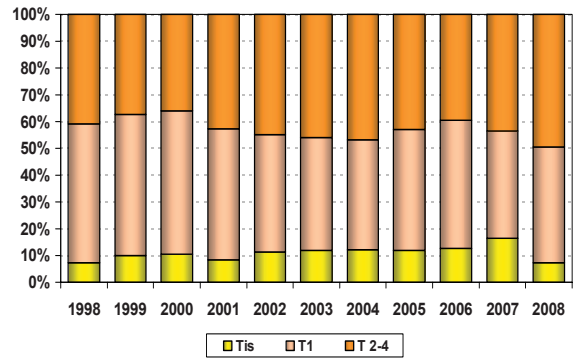


Abb. 4. Operative Therapie: Primäroperationen in der HSK 1996-2008.

Abb. 5. Operative Therapie: Entwicklung Sentinel-Lymphknoten-Biopsie.

Abb. 6. Systemische Therapie: (Neo) adjuvante Chemotherapien $1999-2008$ $(\mathrm{n}=1117)$.
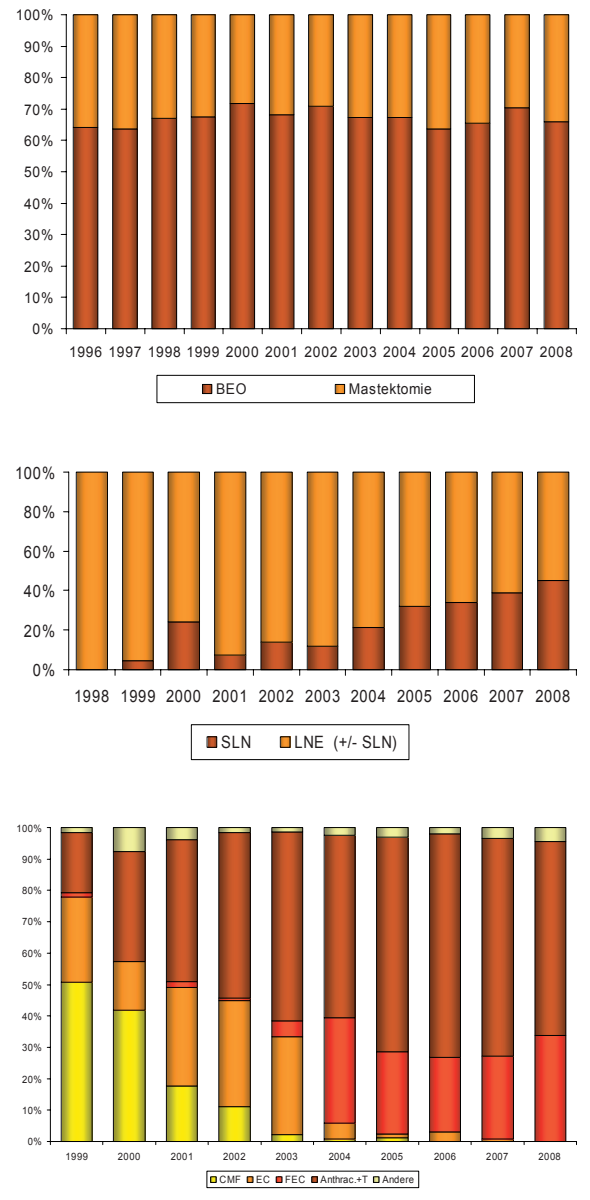

Aber zunächst die Entwicklung des BZ Wiesbaden in Zahlen. Abb. 2 zeigt die seit 1998 kontinuierlich gestiegene Zahl der hier diagnostizierten Mammakarzinome. Trotz der Einführung des Mammographie-Screenings wuchs der Anteil an T2-4-Tumoren im BZ Wiesbaden über die letzten Jahre und erreichte 2008 den höchsten Wert mit etwa 50\% aller Mammakarzinome (Abb. 3). Dieses Phänomen führen wir auf die stabile Kompetenz des Teams und die Anerkennung unter den niedergelassenen Kollegen als Zentrum für komplizierte Fälle zurück.

Trotz der steigenden Zahl von großen Tumoren blieb das Verhältnis von brusterhaltender Therapie zu Ablatio gleich (Abb. 4). Die Erhebung des Axillastatus - die Indikation basiert auf den Leitlinien zur präoperativen Diagnostik - zeigt einen kontinuierlichen Anstieg der alleinigen Sentinellymphknotenbiopsie (Abb. 5). Die konsequente Verfolgung des brusterhaltenden Konzepts wäre nicht ohne Unterstützung der systemischen Therapie möglich.

Am Beispiel des BZ Wiesbaden lässt sich der Paradigmenwechsel in der systemischen Therapie des Mammakarzinoms zeigen (Abb. 6): die führenden chemotherapeutischen Schemata aus dem Jahr 1999 (CMF und EC) wurden im Laufe der Zeit durch das FEC-Schema abgelöst, die Etablierung der taxanhaltigen Chemotherapie lässt sich über Jahre verfolgen, die «anderen» Chemotherapien beinhalten die Regime der zahlreichen adjuvanten und neoadjuvanten Studien, an denen das BZ Wiesbaden beteiligt war. Diese Entwicklungen spiegeln sich in der 5-Jahres-Überlebensstatistik der im BZ Wiesbaden behandelten Patientinnen wider. Mit einer Rate von $83 \%$ im 5-Jahres-Gesamtüberleben positioniert sich das BZ Wiesbaden sowohl national als auch international unter den führenden Zentren auf dem Gebiet «Mammakarzinom».

Um die Versorgung der Patienten weiter zu optimieren, hat die HSK Wiesbaden 2007 einen Antrag auf ambulante Leistungen im Rahmen des §116b, Sozialgesetzbuchs (SGB) V gestellt. Die Grundvoraussetzung war gegeben: Seit Mai 1996 ist die Dr.-Horst-Schmidt-Klinik als Onkologischer Schwerpunkt (OSP-HSK) in Wiesbaden ausgewiesen. Der OSP-HSK reihte sich damit in die Gruppe von etwa 50 Tumorzentren und 70 onkologischen Schwerpunkten ein, die von der Arbeitsgemeinschaft deutscher Tumorzentren in Deutschland anerkannt sind.

Der § 116b war angekündigt als Stärkung der onkologisch tätigen Frauenkliniken im Bereich Gynäkologie, die nun die Karzinompatientinnen von der Diagnostik über Therapie bis zur Nachsorge selbständig in der Klinik mit nur einem Überweisungsschein für 3 Jahre betreuen konnten. Die Verträge werden direkt zwischen Kliniken und Kassen ohne Beteiligung der Kassenärztlichen Vereinigung (KV) geschlossen.

Mit der Klinik für Gynäkologie und Gynäkologische Onkologie HSK Wiesbaden wurde als erste Klinik eine gemeinsame Übereinkunft getroffen, dass die Klinik kein Interesse an einer weiteren ambulanten Tätigkeit hat, die die Interessen der Niedergelassenen berührt. Eine Arbeitsgruppe, bestehend aus dem 2. Vorsitzenden des Bundesvorstandes des Berufsverbands der Frauenärzte e.V. (BVF) und Landesvorsitzenden des BVF Hessen, Herrn Dr. med. König; dem Vorsitzenden des BVF Bezirks Wiesbaden, Herrn Dr. med. Doubek; der stellvertretenden Vorsitzenden des BVF Bezirks Wiesbaden, Frau Dr. Schardt und Klinikdirektor Prof. Dr. med. du Bois sowie seinen Oberärzten mit ambulanter Ermächtigung und der Geschäftsleitung der HSK, hat dieses Konzept realisiert. In einem Brief wurden am 13.08.2008 alle Zu- und Einweiser der Klinik für Gynäkologie und Gynäkologische Onkologie der HSK Wiesbaden über das Konzept informiert. Der Brief war mit den Unterschriften der oben genannten Personen versehen und signalisierte den Konsensus zwischen Klinikern und Niedergelassenen.

In dem Schreiben wurde mitgeteilt, dass die HSK Wiesbaden die Zulassung nach $\$ 116$ b SB V durch das Hessische Sozialministerium bekommen hat. Somit können die Patientinnen mit manifesten Tumorerkrankungen nicht mehr über die persönliche Ermächtigung, sondern im Rahmen der $\S 116$ b-Ambulanz behandelt werden. Dabei sind weder eine Leistungsausweitung noch eine Änderung des therapeutischen Vorgehens oder Angebots vorgesehen. Es werden lediglich formale Änderungen durch Verlagerung der Abrechnung von den KV-Ermächtigungen zur Abrechnung über §116b SGB V stattfinden. 
Praktisch sollte dieser Prozess durch einen Überweisungsschein mit folgenden Vermerken gesteuert werden: 1. Gesicherte onkologische Diagnose und 2. Überweisung in «\$116bAmbulanz». Alle Patientinnen mit Verdachtsdiagnose werden wie bisher in die Ermächtigungsambulanz überwiesen und können nach der Sicherung der Diagnose bei Bedarf in die 116§-Ambulanz überführt werden (Abb. 7).

Es wurde in dem Brief betont, dass der HSK an einem transparenten Verfahren gegenüber den niedergelassenen Kollegen gelegen sei. Als Vertrauensbeweis legt der Klinikchef jährlich seine Klinikabrechnungsunterlagen dem hessischen BVF vor.

Die im Rahmen der Arbeitsgruppe getroffenen freiwilligen Vereinbarungen wurden klar formuliert:

1. Leistungsbringung durch die Klinik für Gynäkologie und Gynäkologische Onkologie der HSK auf quartalsweise Überweisung durch einen gynäkologischen Facharzt/Fachärztin (Gesetzlich gefordert ist lediglich die Überweisung durch einen Vertragsarzt/-ärztin einmal in 3 Jahren).

2. Keine Ausweitung des Spektrums in Bereiche die auch bisher kompetent von den niedergelassenen Gynäkologinnen und Gynäkologen erbracht wurden (z.B. Nachsorge).

3. Transparenz durch Offenlegung der Fallzahl- und Leistungsentwicklung gegenüber den Vertretern des Berufsverbandes auf jährlicher Basis.

Es ist mittlerweile $1 \mathrm{Jahr}$ vergangen, seitdem die HSK die ambulanten Strukturen auf die $\$ 116 b-S c h i e n e$ umgestellt hat. Nach einer Gewöhnungsphase mit etwas organisatorischem Mehraufwand - §116b-Stempel für jeden Schein, der im Haus weiter geleitet wird, eigene Rezepte für jeden Facharzt, der in einem von beiden Zentren (Brustzentrum und Zentrum für Gynäkologische Tumoren) tätig ist, interne Absprachen zwischen Kooperationskliniken usw. - entwickelte sich eine effiziente Struktur. Die diagnostischen Prozesse können rei-
Abb. 7. Algorithmus für den praktischen Ablauf der Überweisung der Patientinnen.

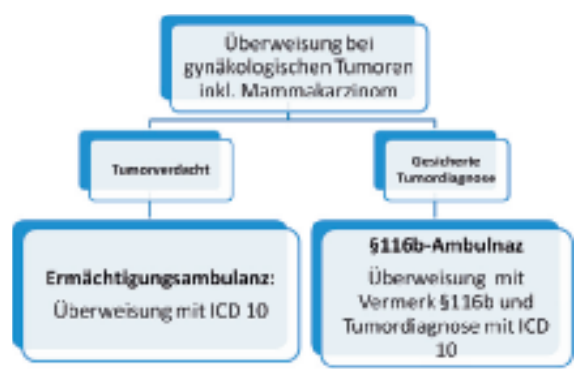

bungsloser abgewickelt werden (z.B. MRT-Mamma), die therapeutischen Konzepte schneller etabliert werden (z.B. das Rezeptieren der supportiven Medikamente im Rahmen einer Chemotherapie), konsiliarische Leistungen jeder Klinik im Haus können problemlos wahrgenommen und abgerechnet werden usw. Die vereinbarten Prozessabläufe werden strikt beachtet. Mögliche Konflikte mit den niedergelassenen Frauenärzten, die weiterhin die Nachsorge und Nachbetreuung bei ihren Patientinnen durchführen, werden dadurch vermieden.

Die Entwicklung dieses Prozesses in Zahlen lässt sich noch schwer abschätzen, da das neue System gerade erst ein Jahr alt ist. Im ersten Quartal 2009 wurden in der §116b-Ambulanz 1925 Patientenbesuche, im zweiten Quartal 1974 und im dritten Quartal 2103 Patientenbesuche registriert. Diese Steigerung führen wir auf das wachsende Vertrauen der niedergelassenen Kollegen in das Konzept zurück.

Neben der Optimierung der Patientenversorgung war das Ziel der neuen Gesetzgebung mit §116b SGB V einen Abrechnungsmodus für hochspezialisierte Leistungen einer Klinikambulanz einzuführen, ohne die KV-Budgets zu belasten. Am Beispiel unserer Erfahrung mit diesem neuen Instrument können wir dies positiv bestätigen. 